\title{
Evaluation in Volunteers of a Candidate Live Oral Attenuated Salmonella typhi Vector Vaccine
}

\author{
David M. Hone, ** Carol O. Tacket, * Andrea M. Harris, * Bradford Kay, * Genevieve Losonsky, a and Myron M. Levine * \\ ${ }^{*}$ Center for Vaccine Development, Division of Geographic Medicine, Department of Medicine, and ${ }^{\ddagger}$ Medical Biotechnology Center, \\ University of Maryland at Baltimore, Baltimore, Maryland 21201
}

\begin{abstract}
Candidate vector vaccine strain CVD $906\left(\right.$ aroC $^{-}$and aroD ${ }^{-}$ derivative of virulent Salmonella typhi strain ISP1820) was evaluated in phase 1 clinical trials. The first nine volunteers ingested a single dose of $5 \times 10^{7} \mathrm{CVD} 906$ bacilli. At this dose CVD 906 stimulates remarkable systemic and mucosal immune responses, inasmuch as $89 \%$ of volunteers developed marked serum antibody levels to $S$. typhi antigens and high numbers of antigen-specific gut-derived antibody-secreting cells. Four (44\%) volunteers developed asymptomatic vaccinemia 4-10 d after immunization and all volunteers excreted CVD 906 on at least one occasion. However, two volunteers developed febrile adverse reactions, one on the day of vaccination and the other on day 4. Of 11 volunteers who ingested a single dose of $5 \times 10^{3}$ CVD 906 bacilli, none displayed side effects but $27 \%$ developed significant serum responses to S. typhi LPS. In vitro, CVD 906 replicates for only nine generations in pooled human serum, indicating that CVD 906 growth is limited in this physiologically relevant medium. In phorbol myristate acetate-induced U937 human macrophage-like cells, CVD 906 replicates intracellularly to a lesser extent than parent strain ISP1820. Although, strain CVD 906 is attenuated and highly immunogenic, the occasional febrile reactions at high doses indicate that further attenuation of this strain is necessary. (J. Clin. Invest. 1992. 90:412-420.) Key words: auxotroph • macrophage $\bullet$ Salmonella • typhoid • U937 monocytes
\end{abstract}

\section{Introduction}

To apply greater control over typhoid fever, which currently results in about 30 million cases and 500,000 deaths each year (1), the World Health Organization has given a high priority to the improvement of vaccines against Salmonella typhi (2). The development of a safe but highly immunogenic live oral typhoid vaccine for use in such programs would have advantages over the currently available reactogenic whole-cell parenteral typhoid vaccines and the well-tolerated but modestly immunogenic live oral typhoid vaccine strain Ty21a (3-5). In addition, a highly immunogenic live oral typhoid vaccine would ideally be suited as a carrier of genes that express protec-

Address reprint requests to Dr. Hone, Center for Vaccine Development, University of Maryland School of Medicine, 10 South Pine Street, Baltimore, MD 21201.

Received for publication 31 May 1991 and in revised form 2 January 1992.

J. Clin. Invest.

(C) The American Society for Clinical Investigation, Inc.

0021-9738/92/08/0412/09 \$2.00

Volume 90, August 1992, 412-420 tive antigens, cloned from other pathogens (6-9). Such hybrid recombinant Salmonella vaccines are expected to invoke protective immunity in humans against both the carrier strain as well as the foreign antigen.

In the 1950s, Bacon and co-workers (10) showed that a $p$-aminobenzoic acid (PABA) ${ }^{1}$-dependent strain of $S$. typhi was attenuated in mice (10). This work did not receive much attention until recently when Hoiseth and Stocker (11) showed that an aroA mutant of Salmonella typhimurium, dependent on aromatic amino acids, PABA, and 2,3-dihydroxybenzoate (DHB; restores ability to synthesize the iron chelator enterochelin) for growth, was attenuated but immunogenic in mice.

Building on this earlier observation, Edwards and Stocker (12) carefully constructed a nonreverting aromatic-dependent mutant (aroA) of $S$. typhi strain CDC 10-80. Because there exists a very remote chance that a single mutation can be lost and the wild-type allele restored as a result of in vivo DNA exchange with other organisms, a nonreverting purA deletion mutation (causing adenine or adenosine dependence) was introduced into their aromatic-dependent derivative for added safety (12). In clinical trials two such $S$. typhi aro $A$ purA strains were remarkably well tolerated by volunteers and elicited cellmediated immunity in nearly all volunteers (4). Unfortunately, $S$. typhi aroA purA strains induced only meager humoral responses in a small percentage of volunteers even when two $10^{9}$-organism doses were given (4). The noneffectiveness of the $S$. typhi aroA, purA mutants suggests that $S$. typhi mutants with only a single auxotrophic requirement should be evaluated as live oral typhoid vaccines.

There are data showing that aro mutants of Salmonella are attenuated in animals but retain high levels of immunogenicity (11, 13-18). This finding, taken together with a study of $S$. typhimurium auxotrophic mutants in mice showing that the purA mutation either alone or in combination with aro $A$ results in poorly immunogenic vaccine strains $(15,18)$, suggests that evaluation of $S$. typhi aro mutants is a direction of choice. The aro genes are well separated on the Salmonella chromosome (19) and several individual aro genes have been shown to be equally attenuating $(12,20-22)$.

\section{Methods}

\section{Bacterial strain and media}

Strain CVD 906, a derivative of wild-type $S$. typhi ISP1820, carries two well-separated, genetically well-defined aro mutations, $\triangle$ aroC 1019 and $\Delta a r o D 1013(22)$. Strains ISP1820 (O9, 12; $\mathrm{Vi}^{+}$, phage type 46; $\mathrm{H}-\mathrm{d})$ and $\mathrm{CVD} 906\left(\mathrm{O} 9,12 ; \mathrm{Vi}^{+} ; \mathrm{H}-\mathrm{d}\right)$ were grown at $37^{\circ} \mathrm{C}$ aerobically on Aro-agar, which includes $0.5 \%$ (wt/ vol) yeast extract (Difco Labora-

1. Abbreviations used in this paper: ASC, antibody-secreting cell; DHB, 2,3-dihydroxybenzoate; PABA, $p$-aminobenzoic acid. 
tories, Inc., Detroit MI), 1\% (wt/vol) Bacto-tryptone (Difco Laboratories, Inc.), $1.5 \%$ Bacto-agar (Difco Laboratories, Inc.), $0.5 \% \mathrm{NaCl}$ (Sigma Chemical Co., St Louis, MO), $0.2 \%$ (wt/vol) casamino acids (Difco Laboratories, Inc.), $0.25 \%$ (wt/vol) glucose (Sigma Chemical Co.), 73 mM PABA, 73 mM DHB (Sigma Chemical Co.), and 100 $\mu \mathrm{g} / \mathrm{ml}$ ferric ammonium citrate (Sigma Chemical Co.), which maximized the growth of CVD906 relative to ISP1820. Liquid cultures were grown at $37^{\circ} \mathrm{C}$ with shaking for $16 \mathrm{~h}$ in Aro-broth which is Aro-agar without the addition of Bacto-agar. Strains were stored in storage medium consisting of an equal volume mixture of $100 \%$ ( $\mathrm{vol} / \mathrm{vol}$ ) glycerol and Aro-broth at $-85^{\circ} \mathrm{C}$.

\section{Growth in human serum}

Pooled normal human sera (Gibco, Grand Island, NY) was tested for $S$. typhi-specific antibody by enzyme-linked immunosorbent assays (ELISA) using $S$. typhi lipopolysaccharide (LPS) or formalin-inactivated $S$. typhi Ty2 cells as antigens (4). Serum substantially free of antibody specific for $S$. typhi, was heat inactivated at $56^{\circ} \mathrm{C}$ for $1 \mathrm{~h}$. To triplicate 2-ml aliquots of serum, $S$. typhi bacilli, grown in $0.8 \%$ (wt/ vol) Nutrient broth (Difco, Inc.) at $37^{\circ} \mathrm{C}$ anaerobically, suspended in $10 \mu \mathrm{l}$ of $N$-saline $(0.9 \% \mathrm{wt} / \mathrm{vol} \mathrm{NaCl})$ were added at a starting viability of 50 colony-forming units (cfu $) / \mathrm{ml}$. Immediately a sample was taken and spread onto Aro-agar and incubated at $37^{\circ} \mathrm{C}$ for $18 \mathrm{~h}$. The remaining serum/cell suspension was placed at $37^{\circ} \mathrm{C}$. Then at $3,6,9$, and $24 \mathrm{~h}$ after inoculation additional samples were taken and viable counts were determined. Results are expressed as the $\log _{10}$ of the arithmetic mean $\mathrm{cfu} / \mathrm{ml}$ of each triplicate $\pm 2 \mathrm{SD}$ versus time in hours.

\section{Growth in U937 cells}

Human histiocytic lymphoma cell line U937 was cultured in RPMI 1640 (Gibco) supplemented with $10 \%$ (vol/vol) fetal calf serum (Gibco) as described (23). U937 cells cultured without antibiotics were stimulated with phorbal myristate acetate (PMA, Sigma Chemical Co.) at a final concentration of $6 \mathrm{ng} / \mathrm{ml}$ for $3 \mathrm{~d}$ before infection and allowed to differentiate into adherent macrophage-like cells (23). Microscopic examination of U937 cells $3 \mathrm{~d}$ after PMA induction revealed high levels of intracellular vacuolation. Each well of a 96-well tray contained $\sim 10^{6}$ adherent $\mathrm{U} 937$ cells. Liquid cultures of $S$. typhi were grown in Aro-broth at $37^{\circ} \mathrm{C}$ with shaking to about $4 \times 10^{8} \mathrm{cfu} / \mathrm{ml}$. The bacterial cells were harvested by centrifugation then washed three times in the U937 culture medium. Dilutions of the bacterial cell suspensions were added to U937-containing culture wells such that the multiplicity of infection was two bacteria per U937 cell. Triplicates for each strain for each time point were cultured in parallel. After addition of the bacteria the plates were centrifuged at $250 \mathrm{~g}$ for $5 \mathrm{~min}$ to bring more bacteria in close contact with the U937 cells and enhance the numbers of bacteria phagocytosed (24). After $1 \mathrm{~h}$ at $37^{\circ} \mathrm{C}$ gentamicin (Sigma Chemical Co.) was added at a final concentration of $200 \mu \mathrm{g} / \mathrm{ml}$ to inactivate extracellular bacteria (24). After $1 \mathrm{~h}$ at $37^{\circ} \mathrm{C}$ with this high level of gentamicin, the cells were washed four times in culture medium and then maintained for the remaining time of the experiment with gentamicin at $10 \mu \mathrm{g} / \mathrm{ml}$ and incubated at $37^{\circ} \mathrm{C} / 5 \% \mathrm{CO}_{2}$. Samples were taken at the time of, and 1 (0-h time point), $9(8 \mathrm{~h})$, and $25(24 \mathrm{~h}) \mathrm{h}$ after, the initial addition of gentamicin as described and bacterial viable counts were determined. The results were plotted graphically as the $\log _{10}$ of the mean of each triplicate \pm 2 SD versus time in hours.

\section{Inocula preparation}

Methods for handling, storage, and inocula preparation of CVD 906 for the clinical trials were strictly in accordance with methods described in an Investigational New Drug Master File reviewed by the Food and Drug Administration. Briefly, a master seed lot of strain CVD 906 was prepared by streaking a single colony isolate of CVD 906 onto an Aroagar plate and incubating at $35^{\circ} \mathrm{C}$ for $18 \mathrm{~h}$. Cells were harvested from this plate by means of a sterile swab, suspended in storage medium, and dispensed as $1-\mathrm{ml}$ aliquots in $2-\mathrm{ml}$ vials. Several vials containing the master seed lot were stored at $-85^{\circ} \mathrm{C}$. Purity was checked by culturing from the frozen master seed lot onto Aro-agar and testing the biotype, serotype, and growth requirements of several single colony isolates. To prepare the inocula the frozen master seed of CVD 906 was steaked onto Aro-agar and incubated for $18 \mathrm{~h}$ at $35^{\circ} \mathrm{C}$. About four single colonies were suspended in ice-chilled phosphate-buffered saline (PBS) then placed on ice. A sample of this suspension was serotyped. The optical density at $660 \mathrm{~nm}\left(\mathrm{OD}_{660}\right)$ of the remaining suspension was measured and the viability was estimated based on previously determined correlation between the viability and the $\mathrm{OD}_{660}$ of a given suspension of CVD 906. The suspension then was diluted such that $1 \mathrm{ml}$ of the bacterial suspension contained a single inoculum. Dilutions of the final suspension were spread onto Aro-Agar for direct determination of the viable count and purity of the inocula.

\section{Clinical microbiological techniques}

All stool samples taken after vaccination were weighed and graded as described (4). Rectal swabs were taken if a volunteer failed to produce a stool in the previous $24 \mathrm{~h}$. Quantitative and qualitative bacteriological screening methods of stool samples were as described (4). Biotyping and serotyping of CVD 906 and clinical isolates were performed as described (4). Gelatin-encapsulated string devices for collecting duodenal fluid containing bile were used as described on days 6,9 , and 13 after vaccination (4). Blood cultures were taken promptly from volunteers who developed temperatures $>38.2^{\circ} \mathrm{C}$. In addition, routine blood cultures $(5 \mathrm{ml})$ were collected on days $5,8,12$, and 15 after vaccination. Blood culture methods were as described (4).

\section{Volunteers}

The 20 volunteers who participated in the clinical evaluation of strain CVD 906, 9 who ingested $5 \times 10^{7}$, and 11 who ingested $5 \times 10^{3}$, were healthy adults, 18-33 yr of age, with no prior history of typhoid fever or typhoid vaccination. They were recruited from the metropolitan Baltimore community and admitted to the isolation ward of the Center for Vaccine Development for $20 \mathrm{~d}$. Preclinical screening and informed consent was obtained from each volunteer (4).

\section{Vaccination and clinical surveilance}

The candidate vaccine strain CVD 906 was administered by the oral route. The volunteers were allowed to acclimatize in the isolation ward for a 48-h period before vaccination and refrained from eating for 90 min before immunization. To neutralize the gastric acid of each volunteer, $2 \mathrm{~g}$ of $\mathrm{NaHCO}_{3}$ were dissolved in $150 \mathrm{ml}$ of sterile distilled water ( $\mathrm{pH} 8.15$ ), and each volunteer ingested $120 \mathrm{ml}$ of the bicarbonate solution. 1 min later, each volunteer ingested the remaining $30 \mathrm{ml}$ of bicarbonate solution mixed with the appropriate inoculum dose of the candidate vaccine strain CVD 906 ( mixed no more than $10 \mathrm{~min}$ before vaccination). The viability of CVD 906 was not affected when CVD 906 was suspended in this bicarbonate solution for up to $30 \mathrm{~min}$ at room temperature (data not shown). The volunteers remained without food and water for $90 \mathrm{~min}$ after ingestion of the inoculum dose.

Clinical surveillance was performed as described (4). The volunteers were interviewed daily after vaccination to determine whether such side effects as fevers, chills, headache, malaise, or diarrhea were experienced. Temperatures, measured orally, were taken at 6-h intervals for $20 \mathrm{~d}$ after vaccination. If a volunteer developed culture-confirmed bacteremia or if their temperature remained elevated $>38.2^{\circ} \mathrm{C}$ for $>48 \mathrm{~h}$, then treatment with oral amoxicillin (a first dose of $1.5 \mathrm{~g}$, then $1 \mathrm{~g}$ every $6 \mathrm{~h}$ for $14 \mathrm{~d}$ ) was begun. Ciprofloxacin ( $500 \mathrm{mg}$ every 12 $h$ for $14 \mathrm{~d}$ ) was given to all volunteers begining on day 12 after vaccination.

\section{Immunological methods}

Sample collecting and processing. Blood samples for serology were collected before and on days 7, 21, and 28 after vaccination. Approximately $70 \mathrm{ml}$ of jejunal fluid was collected and processed before and 15 $\mathrm{d}$ after vaccination by upper intestinal intubation as described (4). Total IgA in the jejunal fluids was measured by ELISA and each specimen was standardized to contain $20 \mathrm{mg}$ of IgA per $100 \mathrm{ml}$ of jejunal fluid (4). PBMC were obtained before and 4, 7, 10, and $14 \mathrm{~d}$ after 
vaccination by centrifugation of $\sim 30 \mathrm{ml}$ of venous blood on FicollHypaque (Organo Teknika, Durham, NC) and processed as described to a final working concentration of $10^{7} \mathrm{PBL} / \mathrm{ml} \mathrm{(25).}$

Antigen preparation. S. typhi LPS was from Difco Laboratories. Vi polysaccharide was purified from $S$. typhi by the method of Wong and Feeley (26). H-d flagella antigen was prepared from $S$. typhi strain 514Ty (12) as described (27).

Serology. IgG, IgM, and IgA immune responses in sera were measured to $S$. typhi LPS, and Vi by ELISAs (4). Net optical densities at $405 \mathrm{~nm}\left(\mathrm{OD}_{405}\right)$ were measured by the differences obtained in serum samples binding to wells coated with antigen minus the $\mathrm{OD}_{405}$ in wells containing sera binding to wells coated only with buffer. Significant LPS-specific IgG seroresponse were defined as those sera that produced a change in net $\mathrm{OD}_{405} \geq 0.2$ (from pre- to post-samples) at a 1:100 dilution. All other antibody class-specific LPS or Vi responses were considered significant if fourfold rises in titer (from pre- to postvaccination samples) were detected using twofold dilutions of sera starting at 1:25. End points were determined using 0.1 as the net $\mathrm{OD}_{405}$ cutoff for LPS-, whole cell-, and H-d antigen-specific serum responses. The net $\mathrm{OD}_{405}$ cutoffs for $\mathrm{Vi}$ antigen-specific serum responses were $\mathbf{0 . 2}$ for IgG, 0.15 for IgA, and 0.3 for IgM.

Widal assays were performed to detect flagella agglutinating antibodies using $S$. virginia (expresses $\mathrm{H}-\mathrm{d}$ flagella) as antigen (4). Also, an ELISA was developed for the evaluation of $S$. typhi $\mathrm{H}$-d-specific antibodies. Cooke polystyrene "U" bottom 96 -well plates were coated with $5 \mu \mathrm{g} / \mathrm{ml}$ antigen in carbonate buffer $(\mathrm{pH} \mathrm{8.0})$ alternating with negative control noncoated wells. Optimal coating concentration was determined by checkerboard titration using a high titered pooled widal-positive (using $S$. virginia $\mathrm{H}-\mathrm{d}^{+}$as the agglutinating antigen) serum specimen. After washing with PBS containing 5\% Tween 20 (PBS-Tween), $\mathrm{pH} 7.2$, plates were blocked with PBS 5\% fetal bovine serum for $1 \mathrm{~h}$ at room temperature. Serum specimens then were added at twofold dilutions starting at $1: 25$ and incubated at $37^{\circ} \mathrm{C}$ for $1 \mathrm{~h}$. After washing and incubating with the appropriate conjugate, the assay was developed with substrate and the plates were read after $30 \mathrm{~min}$. The end-point titration $\mathrm{OD}_{405}$ level was determined by using pooled sera from a negative control population of 30 U.S. volunteers. The mean net $\mathrm{OD}_{405}$ of these control sera +2 SD above the mean was used as the cutoff net $\mathrm{OD}_{405}$; a serum dilution with an $\mathrm{OD}_{405}$ value below the cutoff $\mathrm{OD}_{405}$ was considered not to contain antibody. A fourfold rise in titer from pre- to postsera of the typhoid vaccination study group was considered significant.

Intestinal secretory IgA antibodies to LPS, $\mathrm{H}$, and $\mathrm{Vi}$ antigens were measured by ELISA (4). Fourfold or greater rises in titer were considered significant.

Detection of antibody-secreting cells (ASCs). PBMC were used for the detection of $S$. typhi LPS-, H-d-, and Vi-specific IgG, IgM, and IgA ASC responses by ELISPOT (25).

\section{Results}

\section{Clinical evaluation}

\section{CLINICAL RESPONSE}

Ingestion of $5 \times 10^{7} \mathrm{CVD} 906$ caused febrile responses in two of the nine volunteers in this group. The first febrile response occurred $\sim 24 \mathrm{~h}$ after vaccination when a volunteer (volunteer 13001-4; 117.7-kg female) developed a temperature maximum of $38.4^{\circ} \mathrm{C}$ (Fig. 1 ). This volunteer also experienced abdominal discomfort and a headache but recovered without antibiotic intervention from these early symptoms. On days 7-9 the volunteer reported abdominal discomfort without accompanying headache or malaise and remained afebrile. Again the abdominal symptoms subsided without therapeutic intervention but the abdominal symptoms recurred on day 12 for a further $3 \mathrm{~d}$.

\begin{tabular}{|c|c|c|c|c|c|c|c|c|c|c|c|c|c|c|c|c|c|c|}
\hline Alond & & 0 & & & & & & + & & & 0 & 0 & 0 & 0 & & 0 & & 0 \\
\hline Stool & 4 & + & 0 & - & 0 & 0 & 0 & 0 & 0 & 0 & 0 & 0 & 0 & 0 & 0 & 0 & 0 & 0 \\
\hline
\end{tabular}
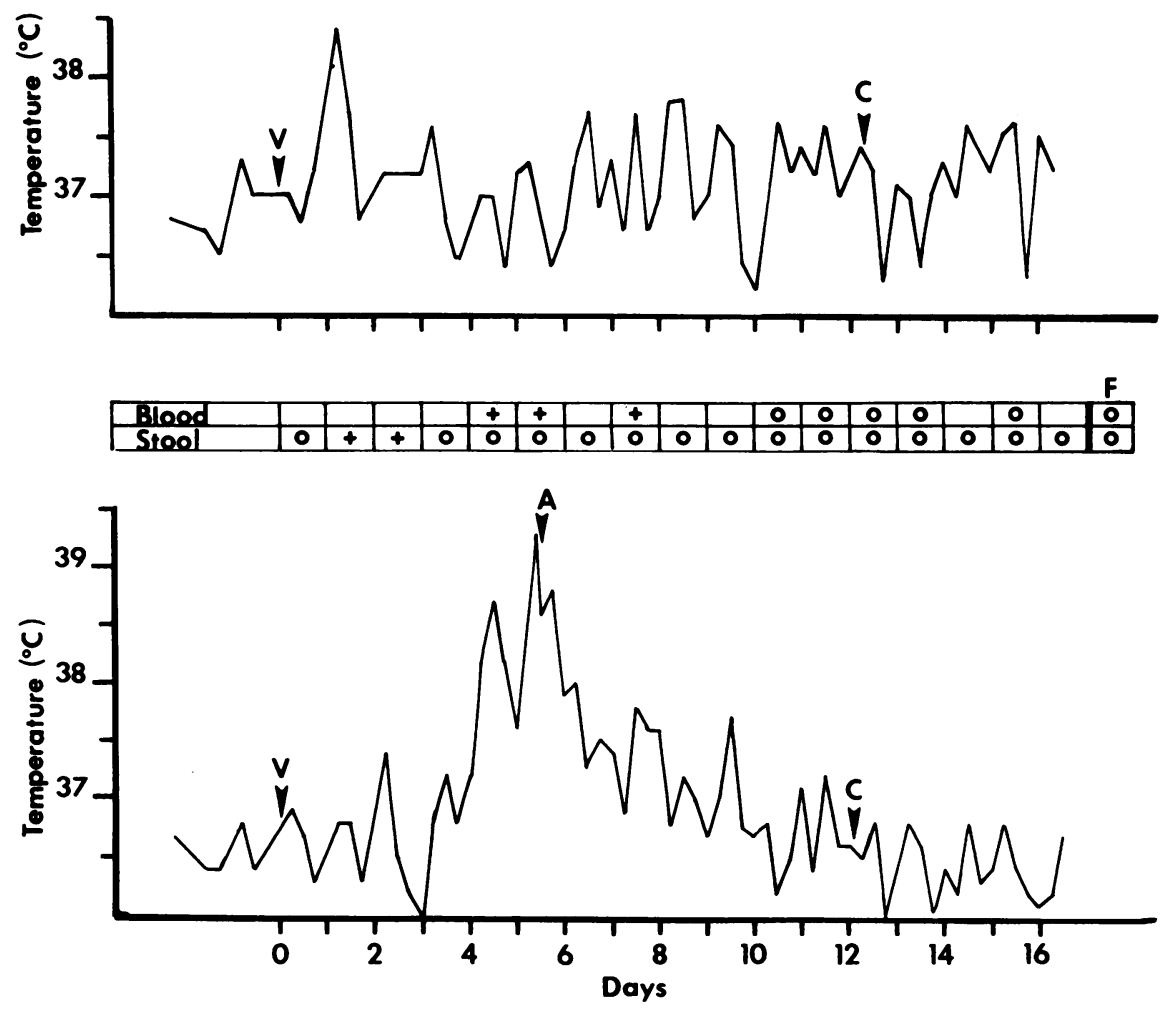

Figure 1. Clinical response of febrile volunteers. The plots of oral temperature $\left({ }^{\circ} \mathrm{C}\right)$, taken every $6 \mathrm{~h}$, of the two febrile volunteers versus time in days after vaccination is shown. The time of vaccination is labeled with the letter $V$. The arrow indicates the time at which antibiotic intervention commenced; $A$, amoxicillin; $C$, ciprofloxacin. The panel above the temperature charts shows the results of the volunteer's blood and stool cultures with each box equivalent to a day; $+=$ positive, $\mathrm{O}=$ negative. $F$ denotes follow-up cultures. 
This volunteer was treated with ciprofloxacin on day 12 after vaccination.

The other febrile reaction began on day 4 , when volunteer $13001-1$ (87.3-kg male) developed an oral temperature of $38.6^{\circ} \mathrm{C}$. The temperature of the volunteer briefly subsided before increasing to a peak of $39.5^{\circ} \mathrm{C}$ (Fig. 1). This febrile volunteer also experienced a headache, nausea, abdominal discomfort, and cough but showed no evidence of toxemia or significant malaise. Antibiotic therapy was initiated $24 \mathrm{~h}$ after onset of this symptom complex after which the volunteer's elevated oral temperature and other symptoms gradually abated (Fig. 1).

At a dose of $5 \times 10^{3}$ cfu $S$. typhi CVD 906 did not cause any notable clinical side effects among the 11 vaccinees.

\section{BACTERIOLOGY}

Excretion and string cultures. After ingestion of $5 \times 10^{7} \mathrm{CVD}$ 906 bacilli, five of nine volunteers $(55.5 \%)$ excreted viable CVD 906 bacilli on the day of vaccination (first $24 \mathrm{~h}$ ). All nine volunteers excreted from $\sim 1 \times 10^{3}$ to $2 \times 10^{6}$ viable CVD 906 bacilli per gram of stool during the period $24-48 \mathrm{~h}$ (day 1 ) after vaccination. Seven of nine volunteers $(77.8 \%$ ) excreted $\sim 1 \times$ $10^{3}$ to $2 \times 10^{5}$ CVD 906 bacilli per gram of stool 48-72 h (day 2 ) after vaccination. Only two of nine volunteers $(22 \%)$ excreted $\sim 2 \times 10^{4}$ viable CVD 906 bacilli $72-96$ h (day 3 ) after vaccination. All the remaining daily coprocultures from each volunteer in this cohort were negative until day 10 when one volunteer passed a single positive coproculture containing $10^{4}$ viable CVD 906 bacilli per gram of stool but no further positive stool cultures were received from this volunteer. Similarly on day 11 after vaccination another volunteer in this cohort passed a single positive coproculture containing CVD 906 bacilli. Insufficient numbers were obtained to provide quantitative estimates, but as with the other case no further positive cultures were isolated from this volunteer.
None of the volunteers in this cohort experienced diarrhea during the course of the study. All duodenal string cultures from volunteers in the high dose cohort, performed on days 6 , 9, and 13 after vaccination, were negative for CVD 906 bacilli.

After ingestion of $5 \times 10^{3}$ CVD 906 bacilli, none of the volunteers excreted detectable CVD 906 bacilli, none of the volunteers experienced diarrhea, and no duodenal string cultures was positive in this group.

Blood cultures. The volunteer who became febrile on day 4 after vaccination was blood culture positive for CVD 906 bacilli at the time of the febrile episode and remained blood culture positive until day 7 (Fig. 1). Also, four afebrile volunteers produced positive blood cultures on day 7 after vaccination. One of this group of afebrile, blood culture-positive volunteers produced a second positive blood culture containing CVD 906 bacilli on day 10 after vaccination. Blood isolates of CVD 906 from the volunteers were stored at $-85^{\circ} \mathrm{C}$. The blood isolates displayed no measurable changes in aromatic growth factor requirements, serotype, and biotype, as compared to that of the parental strain CVD 906.

\section{SEROLOGY}

The majority of volunteers vaccinated with $5 \times 10^{7} \mathrm{cfu}$ of CVD 906 manifested highly significant serum humoral responses specific for $S$. typhi LPS and H-d antigen (Table I). Also Widalpositive sera were found in six of the nine volunteers in this cohort (66.7\%; Table I). No significant rises in serum antibody specific for $\mathrm{Vi}$ antigen were detected (data not shown).

As evidence of intestinal immunopriming, impressive IgGsecreting as well as IgA-secreting ASC responses specific for either LPS or H-d flagella antigen developed in $89 \%$ of this high-dose cohort (Table II). Highly significant elevations of IgM-secreting ASC specific for $S$. typhi LPS antigen and IgMsecreting ASC specific for $\mathrm{H}-\mathrm{d}$ flagella antigen were found in $78 \%$ and $89 \%$ of this high-dose group respectively (data not

Table I. Humoral Responses after Vaccination with $5 \times 10^{7}$ cfu of Strain CVD 906

\begin{tabular}{|c|c|c|c|c|c|c|c|c|c|c|c|c|c|c|c|}
\hline \multirow[b]{3}{*}{ Volunteer } & \multirow{3}{*}{$\begin{array}{l}\text { Clinical } \\
\text { reactions }\end{array}$} & \multicolumn{6}{|c|}{ LPS antibody } & \multicolumn{6}{|c|}{ H-d antibody } & & \\
\hline & & \multicolumn{2}{|c|}{ Serum IgG* } & \multicolumn{2}{|c|}{ Serum IgM* } & \multicolumn{2}{|c|}{ Serum IgA* } & \multicolumn{2}{|c|}{ Serum IgG* } & \multicolumn{2}{|c|}{ Serum IgM* } & \multicolumn{2}{|c|}{ Serum IgA* } & \multicolumn{2}{|c|}{ Widal $^{\ddagger}$ titers } \\
\hline & & Pre & Peak & Pre & Peak & Pre & Peak & Pre & Peak & Pre & Peak & Pre & Peak & Pre & Peak \\
\hline $13001-1$ & $\mathrm{~F}_{4} / \mathrm{B}^{8}$ & 0.11 & $1.83^{+}$ & 80 & $1,280^{\star}$ & 40 & $640^{\star}$ & 400 & $25,600^{\star}$ & $<25$ & $3,200^{\star}$ & 25 & $1,600^{\star}$ & $<20$ & $320^{*}$ \\
\hline-2 & & 0.41 & $1.41^{+}$ & 80 & 160 & 20 & 40 & 400 & 800 & $<25$ & $1,600^{\star}$ & 100 & $800^{\star}$ & $<20$ & $40^{\prime \prime}$ \\
\hline-3 & B & 0.33 & $1.56^{+}$ & 40 & $160^{\star}$ & 20 & $160^{\star}$ & 25 & $1,600^{\star}$ & 25 & $6,400^{\star}$ & 25 & $3,200^{\star}$ & $<20$ & $320^{\prime \prime}$ \\
\hline-4 & $F_{1} / B$ & 0.25 & $1.86^{+}$ & 40 & $320^{\star}$ & 40 & $640^{\star}$ & 50 & $3,200^{\star}$ & $<25$ & $6,400^{\star}$ & 25 & $1,600^{\star}$ & $<20$ & $320^{\prime \prime}$ \\
\hline-5 & & 0.08 & $1.18^{+}$ & 20 & $320^{\star}$ & 40 & $320^{\star}$ & 200 & $3,200^{\star}$ & $<25$ & $200^{\star}$ & 50 & $800^{\star}$ & $<20$ & $40^{*}$ \\
\hline-6 & B & 0.45 & $1.75^{+}$ & 20 & 40 & $<20$ & $160^{\star}$ & 100 & $1,600^{\star}$ & $<25$ & $200^{\star}$ & 50 & $1,600^{\star}$ & $<20$ & $40^{*}$ \\
\hline-7 & & 0.38 & $0.61^{+}$ & 80 & 160 & $<20$ & $<20$ & 800 & 800 & $<25$ & 25 & 50 & 50 & $<20$ & $<20$ \\
\hline-8 & & 0.68 & 0.84 & 320 & $2,560^{\star}$ & 40 & $320^{\star}$ & $<25$ & $800^{\star}$ & $<25$ & $51,200^{\star}$ & $<25$ & $3,200^{\star}$ & $<20$ & $1,280^{\prime \prime}$ \\
\hline-9 & B & 0.38 & $1.44^{+}$ & 40 & $320^{\star}$ & $<20$ & $160^{\star}$ & 800 & $6,400^{\star}$ & 50 & $800^{\star}$ & 50 & $1,600^{\star}$ & $<20$ & $<20$ \\
\hline \multicolumn{2}{|l|}{ Responders (\%) } & \multicolumn{2}{|c|}{$8(89)$} & \multicolumn{2}{|c|}{$6(67)$} & \multicolumn{2}{|c|}{$7(78)$} & \multicolumn{2}{|c|}{$7(78)$} & \multicolumn{2}{|c|}{$8(89)$} & \multicolumn{2}{|c|}{$8(89)$} & \multicolumn{2}{|c|}{$7(78)$} \\
\hline \multicolumn{16}{|l|}{ Mean increase } \\
\hline among responders & & \multicolumn{2}{|c|}{1.03} & \multicolumn{2}{|c|}{10.0} & \multicolumn{2}{|c|}{13.7} & \multicolumn{2}{|c|}{42.3} & \multicolumn{2}{|c|}{662.0} & \multicolumn{2}{|c|}{59.0} & \multicolumn{2}{|c|}{33.7} \\
\hline
\end{tabular}

* Serum IgG, IgM, and IgA responses were measured by ELISA using purified $S$. typhi LPS or purified $S$. typhi H-d flagella as antigens. $S$. typhi LPS-specific IgG responses are expressed in net $\mathrm{OD}_{405}$ units: ${ }^{+}=$significant increase-net $\mathrm{OD}_{40 \text { s }}$ increases of $>0.2$ were significant. All other serum responses are expressed as the inversed of the serum ELISA end-point titers: ${ }^{\star}=$ significant increase-rises of more than fourfold are significant. ${ }^{\ddagger}$ Widal assays were performed with $S$. virginia $(\mathrm{H}-\mathrm{d})$ as the agglutinating antigen and are expressed as the inverse of the end-point titer: " = significant increase-rises of greater than fourfold are significant. ${ }^{8} F_{1}$, febrile reaction day $1 ; F_{4}$, febrile reaction day 4 ; B, blood culture positive. 
Table II. ASC and Jejunal Responses after Vaccination with $5 \times 10^{7}$ cfu of Strain CVD 906

\begin{tabular}{|c|c|c|c|c|c|c|c|c|c|c|}
\hline \multirow[b]{3}{*}{ Volunteer } & \multicolumn{4}{|c|}{ LPS antibody } & \multicolumn{4}{|c|}{ H-d antibody } & \multirow{2}{*}{\multicolumn{2}{|c|}{$\begin{array}{l}\text { LPS antibody } \\
\text { (Jejunal IgA }\end{array}$}} \\
\hline & \multicolumn{2}{|c|}{ IgG ASC* } & \multicolumn{2}{|c|}{ IgA ASC* } & \multicolumn{2}{|c|}{ IgG ASC* } & \multicolumn{2}{|c|}{ IgA ASC* } & & \\
\hline & Pre & Peak & Pre & Peak & Pre & Peak & Pre & Peak & Pre & Peak \\
\hline $13001-1$ & 0 & $>2,000^{\star}$ & 0 & $>2,000^{\star}$ & 0 & $>2,000^{\star}$ & 0 & $>2,000^{\star}$ & $<4$ & $8^{*}$ \\
\hline-2 & 0 & $>2,000^{\star}$ & 0 & $>2,000^{\star}$ & 0 & $>2,000^{\star}$ & 0 & $>2,000^{\star}$ & $<4$ & 4 \\
\hline-3 & 0 & $>2,000^{\star}$ & 0 & $>2,000^{\star}$ & 0 & $>2,000^{\star}$ & 0 & $>2,000^{\star}$ & $<4$ & $16^{*}$ \\
\hline-4 & 0 & $>2,000^{\star}$ & 0 & $>2,000^{\star}$ & 0 & $>2,000^{\star}$ & 0 & $>2,000^{\star}$ & ND & ND \\
\hline-5 & 0 & $>2,000^{\star}$ & 0 & $>2,000^{\star}$ & 0 & $>2,000^{\star}$ & 0 & $>2,000^{\star}$ & $<4$ & $8^{*}$ \\
\hline-6 & 0 & $>2,000^{\star}$ & 0 & $>2,000^{\star}$ & 0 & $>2,000^{\star}$ & 0 & $>2,000^{\star}$ & ND & ND \\
\hline-7 & 0 & $11^{\star}$ & 0 & $12^{\star}$ & 0 & $12^{\star}$ & 0 & $7^{\star}$ & ND & ND \\
\hline-8 & 0 & $>2,000^{\star}$ & 0 & $>2,000^{\star}$ & 0 & $>2,000^{\star}$ & 0 & $>2,000^{\star}$ & ND & ND \\
\hline-9 & 0 & $>2,000^{\star}$ & 0 & $>2,000^{\star}$ & 0 & $>2,000^{\star}$ & 0 & $>2,000^{\star}$ & $<4$ & $<4$ \\
\hline Responders (\%) & \multicolumn{2}{|c|}{$9(100)$} & \multicolumn{2}{|c|}{$9(100)$} & \multicolumn{2}{|c|}{$9(100)$} & \multicolumn{2}{|c|}{$9(100)$} & \multicolumn{2}{|c|}{$3(60)$} \\
\hline \multicolumn{11}{|l|}{ Mean increase } \\
\hline among responders & \multicolumn{2}{|c|}{-} & \multicolumn{2}{|c|}{ - } & \multicolumn{2}{|c|}{-} & \multicolumn{2}{|c|}{-} & \multicolumn{2}{|c|}{5.3} \\
\hline
\end{tabular}

* Peripheral blood IgG and IgA ASC were measured using the ELISPOT assay (25) with either $S$. typhi LPS or purified $S$. typhi H-d, as antigens. The results are expressed as the number of ASC $/ 10^{6} \mathrm{PBMC}^{*}=$ increases of $>4 \mathrm{ASC} / 10^{6} \mathrm{PBMC}$ are significant. ${ }^{\ddagger}$ Jejunal IgA responses to $S$. typhi LPS were measured by ELISA and results are expressed as the inverse of the ELISA end-point titer; ${ }^{\star}=$ rises of greater than fourfold are significant.

shown). Also significant jejunal IgA responses manifested in three of five volunteers of the high-dose cohort (Table II).

The serum humoral immune responses elicited by CVD 906 after volunteers ingested $5 \times 10^{3}$ CVD 906 bacilli are shown (Table III). None of this low-dose cohort developed significant rises in serum IgM or IgA specific for $S$. typhi LPS and no significant IgG, IgM, or IgA serum responses specific for H-d flagella antigen were detected by ELISAs (not shown).

Some of the volunteers in the low-dose cohort developed rises in peripheral blood ASC specific for $S$. typhi LPS antigen and H-d flagella antigen after vaccination with $5 \times 10^{3} \mathrm{cfu}$ of CVD 906 (Table III).

\section{Growth in human serum}

Strain CVD 906 grew for about nine generations in pooled human serum whereas parent strain ISP1820 grew through many more generations in pooled human sera (Fig. 2). Adding PABA to human serum at a final concentration of $5.0 \mu \mathrm{M}$ did not significantly increase or decrease the growth rate of, or number of generations achieved by, CVD 906 in pooled hu-

Table III. Humoral and ASC Responses after Vaccination with $5 \times 10^{3}$ cfu of Strain CVD 906

\begin{tabular}{|c|c|c|c|c|c|c|c|c|c|c|}
\hline \multirow[b]{3}{*}{ Volunteer } & \multicolumn{6}{|c|}{ LPS antibody } & \multicolumn{4}{|c|}{ H-d antibody } \\
\hline & \multicolumn{2}{|c|}{ Serum IgG* } & \multicolumn{2}{|c|}{ IgG ASC } & \multicolumn{2}{|c|}{ IgA ASC } & \multicolumn{2}{|c|}{ IgG ASC } & \multicolumn{2}{|c|}{ IgA ASC ${ }^{\ddagger}$} \\
\hline & Pre & Peak & Pre & Peak & Pre & Peak & Pre & Peak & Pre & Peak \\
\hline $13002-1$ & 0.27 & 0.21 & 0 & 0 & 0 & 0 & 0 & 0 & 0 & 0 \\
\hline-2 & 0.81 & $1.02^{+}$ & 0 & 2 & 0 & $4^{\star}$ & 0 & 0 & 0 & 0 \\
\hline-3 & 0.11 & 0.19 & 0 & 0 & 0 & 0 & 0 & 0 & 0 & 0 \\
\hline-4 & 0.31 & 0.31 & 0 & 0 & 0 & 0 & 0 & 0 & 0 & 0 \\
\hline-5 & 0.36 & 0.55 & 0 & 0 & 0 & $49^{\star}$ & 0 & 0 & 0 & 0 \\
\hline-6 & 0.34 & $0.92^{+}$ & 0 & $41^{\star}$ & 0 & $1,106^{\star}$ & 0 & 0 & 0 & $5^{\star}$ \\
\hline-7 & 0.78 & 0.78 & 0 & 0 & 0 & 0 & 0 & 0 & 0 & 0 \\
\hline-8 & 0.78 & 0.78 & 0 & 0 & 0 & 0 & 0 & 0 & 0 & 0 \\
\hline-9 & 0.51 & 0.51 & 0 & 0 & 0 & 0 & 0 & 0 & 0 & 0 \\
\hline-10 & 1.41 & $1.71^{+}$ & 0 & 0 & 0 & 0 & 0 & 0 & 0 & 2 \\
\hline-11 & 0.29 & 0.29 & 0 & 0 & 0 & 0 & 0 & 0 & 0 & 0 \\
\hline Responders (\%) & & & & & & & & & & \\
\hline \multicolumn{11}{|l|}{ Mean increase } \\
\hline among responders & \multicolumn{2}{|c|}{0.32} & \multicolumn{2}{|c|}{ - } & \multicolumn{2}{|c|}{ - } & \multicolumn{2}{|c|}{ - } & \multicolumn{2}{|c|}{ - } \\
\hline
\end{tabular}

* Serum IgG responses shown were measured by ELISA using $S$. typhi LPS as antigen and are expressed as net OD $_{405}$ units: ${ }^{*}=$ significant increase-net $\mathrm{OD}_{405}$ increases of $>0.2$ were significant. ${ }^{\ddagger}$ Peripheral blood IgG and IgA ASC were measured using the ELISPOT assay (25) with either $S$. typhi LPS or purified $S$. typhi H-d as antigens. The results are expressed as numbers of ASC $/ 10^{6}$ PBMC: ${ }^{\star}=$ significant increase-increases of $>4 \mathrm{ASC} / 10^{6} \mathrm{PBMC}$ were significant. 


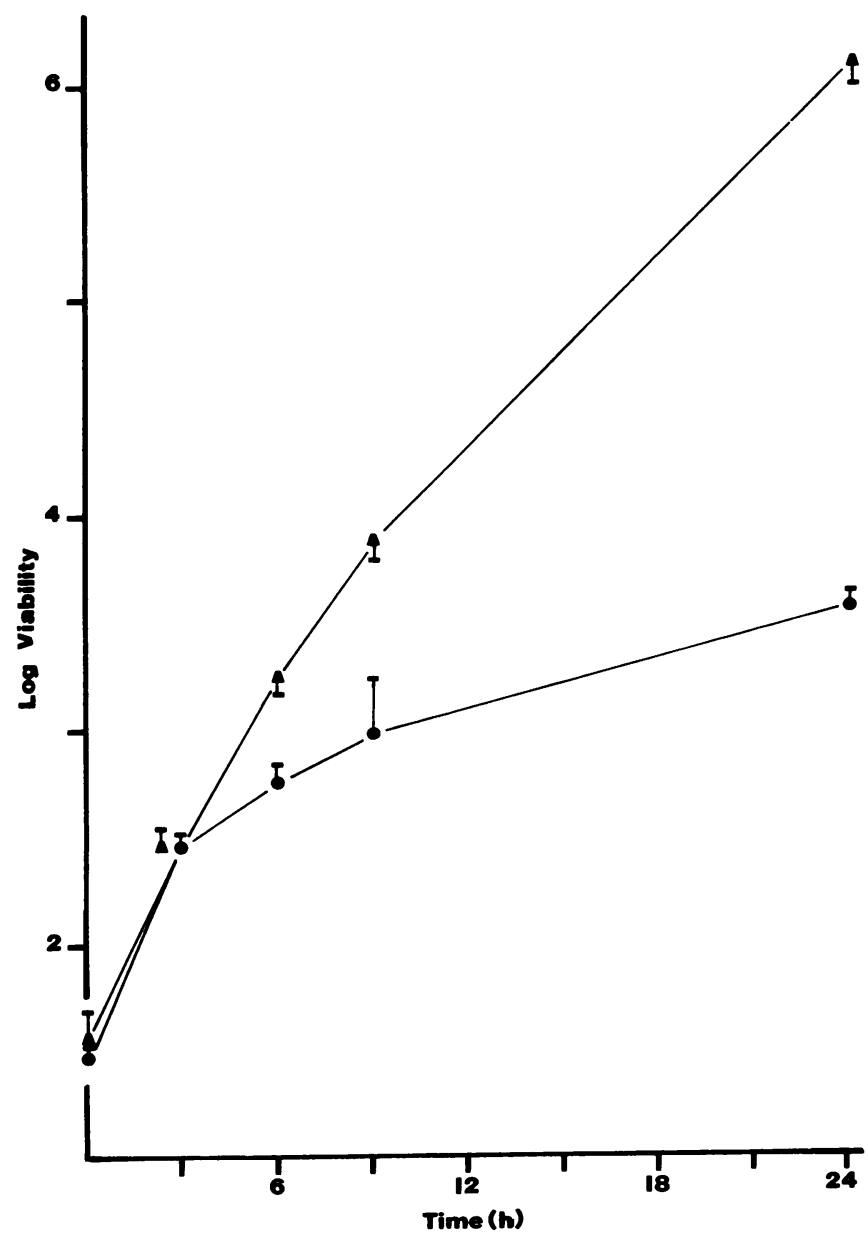

Figure 2. Serum growth curves. The growth curves of $S$. typhi strains ISP1820 $(\Delta)$ and CYD $906(0)$ in pooled human sera are shown in this figure. Each point represents the arithmatic mean of $\log _{10}$ viability $/ \mathrm{ml}$ of triplicate samples $\pm 2 \mathrm{SD}$ at a given time in hours. The data shown are representative of duplicate experiments.

man serum (not shown). The minimum concentration of PABA required to restore maximum growth rate of CVD 906 on minimal media was between 5.0 and $10.0 \mathrm{nM}$; below this concentration (but above $0.05 \mathrm{nM}$ ), CVD 906 replicates but at a markedly reduced rate (data not shown). The minimum concentration of PABA required for growth of the blood isolates of CVD 906, was not measurably altered when compared to CVD 906 (data not shown).

\section{Growth in U937 human macrophages}

The growth of ISP1820 and CVD 906 in U937 cells is shown (Fig. 3). Of $\sim 10^{6}$ viable bacilli of $S$. typhi ISP1820 added to each well containing $\sim 10^{6}$ U937 cells, $4.12 \%$ remained viable $1 \mathrm{~h}$ after addition of gentamicin (i.e., the initial intracellular viable count). A 3.4-fold lower percentage of viable CVD 906 bacilli $(1.22 \%)$ was recovered $1 \mathrm{~h}$ after gentamicin exposure. This lower initial intracellular viable count of CVD 906 was reproduced in two independent experiments. The number of viable ISP1820 and CVD 906 bacilli recovered from U937 cells increases with time but ISP1820 increases at a rate 1.4-fold faster than CVD 906 (Fig. 3). The viability of U937 cells cultured with either ISP1820 or CVD 906 was comparable throughout the experiment.

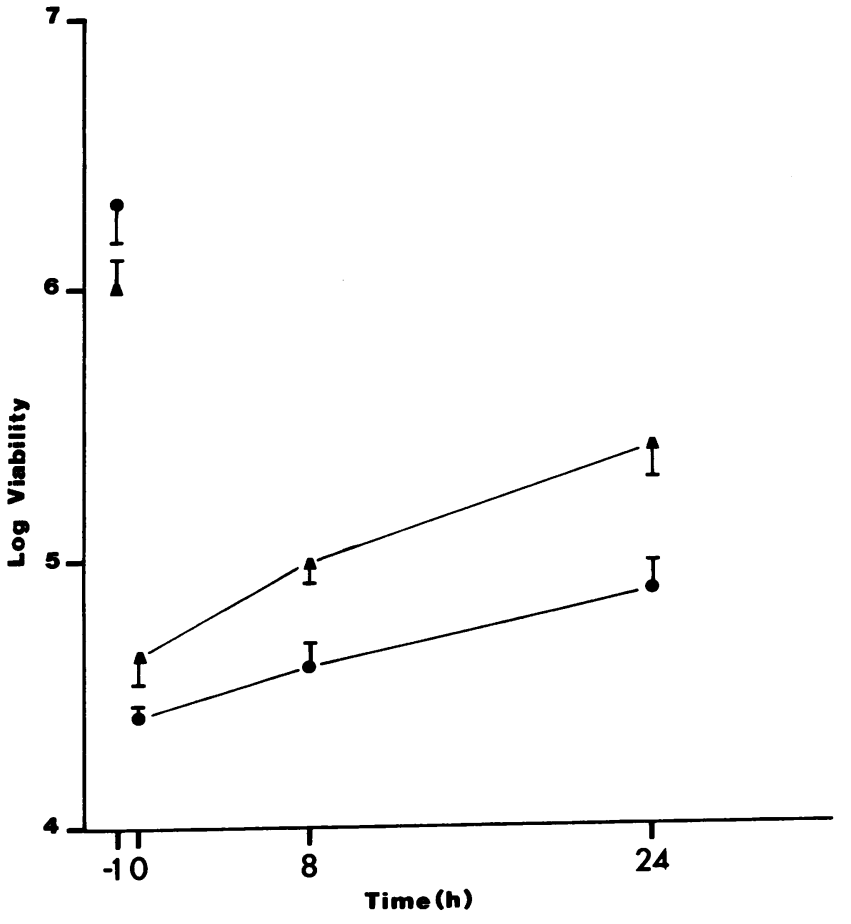

Figure 3. U937 macrophage growth curves. This figure shows the growth characteristics of ISP1820 ( $\triangle$ ) and CVD $906(0)$ after ingestion by PMA-induced U937 human macrophage-like cells. Gentamicin $(200 \mu \mathrm{g} / \mathrm{ml})$ was added just after the -1 -h time point to kill extracellular bacteria. At the 0 time point the high level gentamicin was washed away and for the remainder of the experiment the concentration of gentamicin was maintained at $10 \mu \mathrm{g} / \mathrm{ml}$. Each point represents the arithmatic mean of triplicate samples expressed as $\log _{10}$ viability of bacteria per well $\pm 2 \mathrm{SD}$ at a given time in hours. The data shown are representative of triplicate experiments.

\section{Discussion}

The shortfall of other attenuated $S$. typhi strains $27 \mathrm{~V}$, Ty21a, and $541 \mathrm{Ty}(\operatorname{aroA}$ purA $)$ is their variable or poor immunogenicity $(4,5,28,29)$. However, these strains are remarkably safe such that even after volunteers ingest as many as $10^{10}$ viable $27 \mathrm{~V}$, Ty21a, or 541Ty bacilli no significant side reactions are observed $(4,28,29)$. Clearly, new candidate typhoid vaccines will be judged against this background and will not be considered advantageous over these existing strains unless they achieve similar safety in humans but with greatly enhanced immunogenicity.

A single dose of $5 \times 10^{3}$ CVD 906 bacilli meets this criterion. In placebo-controlled field trials the rate of IgG seroconversion to $S$. typhi LPS among Chileans correlates with protection (28). If this correlate holds true for individuals immunized with CVD 906, then we expect that a single dose of 5 $\times 10^{3}$ CVD 906 bacilli might induce $\sim 25 \%$ protection.

In a strict sense it is difficult to accurately determine the measure of attenuation of CVD 906 in humans, without data from volunteer studies that document the virulence of wildtype parental strain ISP1820. Some measure of the attenuating effect of aro can be deduced by comparing the clinical response of volunteers who ingested CVD 906 with the clinical response of volunteers who ingested virulent $S$. typhi Quailes strain which has a well-documented virulence in humans (29-31). 
Without bicarbonate as buffer, Quailes strain caused fullblown typhoid fever, defined as a temperature $\geq 39.5^{\circ} \mathrm{C}$ for a period of $>36 \mathrm{~h}$, in $0 \%$ of volunteers who ingested $10^{3}$ viable bacilli, in $28 \%$ of volunteers who ingested $10^{5}$ viable bacilli, in $50 \%$ of volunteers who ingested $10^{7}$ viable bacilli, and in $95 \%$ of volunteers who ingested $10^{9}$ viable bacilli (30). In subsequent volunteer challenge studies performed in an identical manner, ingestion of $10^{5}$ viable Quailes bacilli caused typhoid fever in $50 \%$ of unimmunized recipients $(29,31)$. Thus Quailes strain when given without bicarbonate as buffer causes full-blown typhoid fever in $50 \%$ of volunteers who ingest between $10^{5}$ and $10^{7}$ viable Quailes bacilli. It is assumed that the rate of attack in volunteers after ingestion of $10^{5}$ to $10^{7}$ Quailes bacilli would increase to as much as $90-100 \%$ if bicarbonate buffer preceded challenge. If the virulence of $S$. typhi Quailes strains in humans is similar to that of ISP1820, then we would expect full-blown typhoid fever in $\sim 50 \%$ of volunteers who ingest $5 \times 10^{7}$ viable ISP1820 bacilli without bicarbonate and as many as $90-100 \%$ of volunteers when the same dose is given with bicarbonate. However, the clinical response of CVD 906 is notably ameliorated when compared to that of wild-type $S$. typhi infection in humans. Only one of the volunteers (11\%) who ingested $5 \times 10^{7}$ CVD 906 bacilli with bicarbonate may have gone on to meet the definition of full-blown typhoid fever described by Hornick et al. (30), if antibiotic had not been used. Furthermore, asymptomatic bacteremia occurs at a high frequency after ingestion of candidate vaccine strain CVD 906 whereas wild-type $S$. typhi infections rarely cause asymptomatic bacteremias in volunteers (32). We conclude that CVD 906 is considerably attenuated. A more precise measurement of the attenuating effect of the aro mutation will require the evaluation of a double aro mutant of a wild-type $S$. typhi strain that has a well-documented virulence in humans.

Dragunsky et al. (33) reported that $S$. typhi strains Ty21a and 541 Ty (aroA purA) with documented safety in humans are incapable of intracellular growth in U937 monocytes, whereas a galE Vi-negative strain, EX462, that retains some virulence for humans underwent limited growth in these monocytes. Thus, the capacity to grow in U937 monocytes correlates with the documented clinical tolerance of these strains in volunteers. We demonstrated that CVD 906 retains some capacity to grow inside phorbol myristate acetate-induced U937 human macrophage-like cells. Intracellular growth by CVD 906, wherein CVD 906 mainly resides during vaccination, might in part contribute to the occasional adverse clinical reactions in humans.

To account for the attenuating affect of the aro mutation seen in mice, more importance was placed on the PABA dependence of these mutants (34). Milhous et al. (35) reported that only low levels of PABA (1.0-10.0 nM) are present in human serum. Also, intracellular levels are probably lower or equally low as the serum PABA concentration in that mammals acquire the essential vitamin folate from their diet and mammalian cells do not possess the biochemical pathways or require the synthesis and utilization of $\mathrm{PABA}$ for folate production (34). Because the concentration of PABA normally present in mammalian tissue is minute, it has been assumed that, once within mammalian tissue, Salmonella aro mutants rapidly deplete their intracellular pool of PABA and cease to grow, resulting in the observed attenuation of these strains in mice $(11,20)$. In support of this thesis, Hoiseth (36) found that the virulence of an $S$. typhimurium aro mutant in mice was signifi- cantly increased if the mice were maintained on water supplemented with 73 mM PABA, presumably as a result of increasing the serum PABA concentration.

We found that the growth rate of aro mutant CVD 906 was not significantly decreased in minimal medium supplemented with as little as 5.0-10.0 nM PABA. Additionally, CVD 906 is capable of replicating (at reduced growth rates) in minimal media supplemented with minute concentrations of PABA (between 0.05 and $5.0 \mathrm{nM}$ ). Thus, the concentration of PABA required to supplement the growth of CVD 906 is within the range reported in human serum (35), and human serum PABA concentration may be sufficient to supplement the PABA requirement of CVD 906. Indeed, although CVD 906 does not replicate for more than a single generation in the complete absence of exogenous PABA, this candidate vaccine strain replicates for about nine generations in pooled human serum. Furthermore, addition of PABA to human serum did not increase the number of generations achieved by CVD 906 in human serum, suggesting that other factors limit the growth of CVD 906 in serum before exhaustion of PABA in this medium. As such the limited capacity of CVD 906 to replicate in human serum appears to be a consequence of the absence of other growth factors such as an iron chelator, or $p$-hydroxybenzoate.

The importance of the known siderophore defect in S. typhi aro mutants, an inability to synthesize enterochelin, in the attenuation of these mutants was thought to be minimal based on evidence from animal models (34). Benjamin et al. (37) showed that $S$. typhimurium ent mutants, which cannot synthesize enterochelin (an iron chelating catechol), retained full virulence in mice, and concluded that enterochelin is not a virulence factor of $S$. typhimurium in mice. If in fact the inability to synthesize a siderophore such as enterochelin is the dominant growth-limiting factor in vivo that causes the attenuation of CVD 906 in humans, then this might indicate a difference between the phathogenesis of S. typhi in humans and the pathogenesis of $S$. typhimurium in mice. We are currently further investigating this question and how $S$. typhi aro mutants replicate intracellularly, where different growth conditions prevail.

CVD 906 underwent limited growth in individual sera derived from each of the volunteers, suggesting that growth-supplementing metabolites were present in the sera. Hence, growth in human serum per se does not completely explain the occurrence of occasional febrile responses after vaccination. The individuals who developed febrile adverse reactions to CVD 906 might have lower natural immunity (38) or greater sensitivity to $S$. typhi LPS-induced febrile responses $(39,40)$ or be influenced by genetic factors that otherwise increased their sensitivity to $S$. typhi (38). Alternatively, the reactions caused by CVD 906 might be the result of special properties of this strain, whereas other $S$. typhi aro mutants may behave differently in volunteers. Possible further attenuation might derive from mutations that reduce growth rate in vitro (such as $r e c A$ or $c y a-c r p)(41,42)$, or mutations that diminish macrophage resistance (e.g., phoPQ, pag, prg, htrA, or groEL) (43-47). Increasing the phagocytic index of CVD 906 by introducing mutations that block Vi antigen synthesis $(48,49)$, might also produce a further attenuated typhoid vaccine candidate. Vinegative strains have the added advantage of being more sensitive to the bactericidal action of complement thus reducing their growth potential in the extracellular compartment $(3,50)$. 
As a caveat, the clinical experience with CVD 906 once again highlights the limitations of animal models used for assessing Salmonella strains as noted earlier ( 3 ). In calves, a transient increase in rectal temperature of $1.2^{\circ} \mathrm{C}$ on average, was recorded $\sim 1 \mathrm{~d}$ after oral vaccination with $\sim 1.5 \times 10^{11} \mathrm{cfu}$ of S. typhimurium aro mutant strain SL3261 (16); the calves regained normal body temperature and remained healthy without antibiotic or antipyretic treatment. On the other hand, in mice S. typhimurium aro mutant strain SL3261 is regarded as being safely attenuated (12). Because death is used for the mouse typhoid model, in place of, not body temperature as the criterion of virulence, it is possible that a transient febrile response occurs after oral vaccination of mice with aro mutants of $S$. typhimurium. In this perspective, the calf $S$. typhimurium model was more predictive of the outcome in humans. The mouse typhoid model might be improved, however, by including the measurement of body temperature along with lethalities, thereby including experimental criteria more relevant for human vaccine development. Also, in vitro assays such as were performed here provide helpful preclinical data by which attenuated $S$. typhi strains can be evaluated.

In previous clinical and field studies with attenuated $S . t y-$ phi vaccine strain $\mathrm{Ty} 21 \mathrm{a}$, the serum $\mathrm{IgG} \mathrm{O}$ antibody response (measured by ELISA) was found to correlate with the protective efficacy of different immunization regimens as determined in large-scale field trials of vaccine efficacy (28). For this reason, the IgG-ELISA $O$ antibody response has been used as a practical, helpful, and economical measure of the relative immunogenicity of candidate live oral typhoid vaccines. In this context, CVD 906 is impressively immunogenic when compared to previous live typhoid vaccines $(4,28,29)$. To further characterize the specificity of the $S$. typhi LPS responses, sera from the volunteers who ingested $5 \times 10^{7} \mathrm{CVD} 906$ bacilli were assayed for rises in IgG, IgM, and IgA specific for hot phenolpurified $S$. thompson LPS (O-antigen 6, 7; not shown). Two of the nine individuals manifested approximately fourfold rises in serum IgG specific for $S$. thompson LPS but there was no elevation of IgM and IgA titers specific for $S$. thompson LPS. Therefore, the LPS responses shown here are highly specific for $S$. typhi O-antigen.

There were no significant rises in serum antibodies to the $\mathrm{Vi}$ antigen. This lack of responsiveness of the Vi component of live typhoid vaccines has been observed (51). Also, responses to Vi antigen are uncommon after acute typhoid fever; rather, when high titers of antibody to $\mathrm{Vi}$ antigen are seen they are usually associated with the chronic typhoid carrier state (52). In contrast, the Vi antigen, when purified and innoculated parenterally, is highly immunogenic (53), indicating that the poor response to $\mathrm{Vi}$ antigen after vaccination with $\mathrm{Vi}$-positive live typhoid vaccine strains might be the result of low level or transient expression of this antigen in vivo.

Live oral attenuated $S$. typhi strains have potential as carriers of antigen from bacterial pathogens such as enterotoxigenic Escherichia coli (7) or Vibrio cholerae (54), or antigens from viral pathogens such as human immunodeficiency virus (T. Fouts, G. A. Lewis, and D. M. Hone, manuscript in preparation), which attach to, or enter the human host at intestinal mucosal sites. It follows that such live oral $S$. typhi vector vaccines must elicit effective intestinal secretory IgA responses. The ASC assay data presented here show that CVD 906 elicits high levels of intestinal priming of B cells $(27,55,56)$. In fact, the number of ASC detected by ELISPOT ( $>2,000 \mathrm{ASC} / 10^{6}$
PBMC) after immunization with $5 \times 10^{7} \mathrm{CVD} 906$ bacilli is an order of magnitude higher than the number normally seen after vaccination with multiple doses of live oral typhoid vaccine Ty21a (typically up to $\left.100 \mathrm{ASC} / 10^{6} \mathrm{PBMC}\right)(55,56)$.

Although CVD 906 is reactogenic in humans at high doses, CVD 906 retains the important property of immunogenicity. We believe that only modest attenuation of this strain is required to produce a safe and highly immunogenic live oral typhoid vaccine and antigen carrier strain.

\section{Acknowledgments}

We thank Robert Hall and David Maneval for their assistance with preparing $S$. typhi LPS, Vi, and flagella antigens. Also our thanks and appreciation go to Sylvia O'Donnell, the Clinical Microbiology team, and the hospital staff.

This work was supported in part by National Institutes of Health grant NO1-Al-62528. David Hone was supported by the Medical Biotechnology Center.

\section{References}

1. Institute of Medicine. 1986. Prospects for immunizing against Salmonella typhi. In New Vaccine Development: Establishing Priorities. National Academy Press, Washington, DC. Appendix D-14, 1-10.

2. World Health Organization. 1987. In Biomedical and Epidemiological Research Priorities of Global Scientific Working Groups. WHO Publication WHO/ CDD/RES86.8 rev. 1 .

3. Hone, D. M., S. R. Attridge, B. Forrest, R. Morona, D. Daniels, J. T. LaBrooy, R. C. Bartholomeusz, D. J. Shearman, and J. Hackett. 1988. A galE, via ( $\mathrm{Vi}$ antigen-negative) mutant of Salmonella typhi $\mathrm{Ty} 2$ retains virulence in humans. Infect. Immun. 56:1326-1333.

4. Levine, M. M., D. Herrington, J. R. Murphy, J. G. Morris, G. Losonsky, B. Tall, A. A. Lindberg, S. Svenson, S. Baquar, M. F. Edwards et al. 1987. Safety, infectivity, immunogenicity, and in vivo stability of two attenuated auxotrophic mutant strains of Salmonella typhi, 541Ty and 543Ty, as live oral vaccines in humans. J. Clin. Invest. 79:888-902.

5. Levine, M. M., D. M. Hone, B. A. D. Stocker, and M. Cadoz. 1990. Vaccines to prevent typhoid fever. In New Generation Vaccines. G. Woodrow and M. M. Levine, editors. Marcel Dekker, Inc., New York. 269-287.

6. Formal, S. B., L. S. Baron, D. J. Kopecko, O. Washington, C. Powell, and C. A. Life. 1981. Construction of a potential bivalent vaccine strain: introduction of Shigella sonnei form I antigen genes into the galE Salmonella typhi Ty21a vaccine strain. Infect. Immun. 34:746-750.

7. Clements, J. D., and S. El-Morshidy. 1984. Construction of a potential live oral bivalent vaccine for typhoid fever and cholera-Escherichia coli-related diarrheas. Infect. Immun. 46:564-569.

8. Aggarwal, A., S. Kumar, R. Jaffe, D. Hone, M. Gross, and J. Sadoff. 1990 Oral Salmonella: malaria circumsporozoite recombinants induce specific CD8 ${ }^{+}$ cytotoxic T cells. J. Exp. Med. 172:1083-1090.

9. Wu, J. Y., S. Newton, A. Judd, B. Stocker, and W. S. Robinson. 1989. Expression of immunogenic epitopes of hepatitis B surface antigen with hybrid flagellin proteins by a vaccine strain of Salmonella. Proc. Natl. Acad. Sci. USA. 86:4726-4730.

10. Bacon, G. A., T. W. Burrows, and M. Yates. 1950. The effects of biochemical mutation on the virulence of Bacterium typhosum: the virulence of mutants. Br. J. Exp. Pathol. 31:714-724.

11. Hoiseth, S. K., and B. A. D. Stocker. 1981. Aromatic-dependent Salmonella typhimurium are non-virulent and effective as live vaccines. Nature (Lond.). 291:238-239.

12. Edwards, M. F., and B. A. D. Stocker. 1988. Construction of delaroA his del pur strains of Salmonella typhi. J. Bacteriol. 170:3991-3995.

13. Stocker, B. A. D. 1988. Auxotrophic Salmonella typhi as live vaccine. Vaccine. 6:141-145.

14. Eisenstein, T. K., L. M. Killar, B. A. D. Stocker, and B. M. Sultzer. 1984. Cellular immunity induced by avirulent Salmonella in LPS-defective $\mathrm{C} 3 \mathrm{H} / \mathrm{HeJ}$ mice. J. Immunol. 133:958-961.

15. O'Callaghan, D., D. Maskell, F. Y. Liew, C. S. F. Easmon, and G. Dougan. 1988. Characterization of aromatic- and purine-dependent Salmonella typhimurium: attenuation, persistence, and ability to induce protective immunity in Balb/ c mice. Infect. Immun. 56:419-423.

16. Smith, B. P., M. Reina-Guerra, B. A. D. Stocker, S. K. Hoiseth, and E. H Johnson. 1984. Vaccination of calves against Salmonella dublin with aromaticdependent Salmonella typhimurium. Am. J. Vet. Res. 45:1858-1861.

17. Smith, B. P., M. Reina-Guerra, S. K. Hoiseth, B. A. D. Stocker, F. Haba- 
sha, E. Johnson, and F. Merritt. 1984. Aromatic-dependent Salmonella typhimurium as modified vaccines for calves. Am. J. Vet. Res. 45:59-66.

18. Sigwart, D. F., B. A. D. Stocker, and J. D. Clements. 1989. Effect of a purA mutation on efficacy of Salmonella live-vaccine vectors. Infect. Immun. 57:18581861.

19. Sanderson, K. E., and J. A. Hurley. 1984. Linkage map of Salmonella typhimurium. In Escherichia coli and Salmonella typhimurium. Cellular and Molecular Biology. F. Neidhardt, J. L. Ingraham, K. B. Low, B. Magasanik, M. Schaechter, and H. E. Umbarger, editors. American Society for Microbiology, Washington, DC. 877-918.

20. Dougan, G., S. Chatfield, D. Pickard, J. Bester, D. O'Callaghan, and D. Maskell. 1988. Construction and characterization of vaccine strains of Salmo nella harboring mutations in two different aro genes. J. Infect. Dis. 158:13291335.

21. Miller, I. A., S. Chatfield, G. Dougan, L. De Silva, H. S. Joysey, and C. E. Hormaeche. 1989. Bacteriophage P22 as a vehicle for transducing cosmid gene banks between smooth strains of Salmonella typhimurium: use in identifying a role for $a r o D$ in attenuating virulent Salmonella strains. Mol. Gen. Genet. 215:312-316.

22. Hone, D. M., A. M. Harris, S. Chatfield, G. Dougan, and M. M. Levine. 1991. Construction of genetically defined double aro mutants of Salmonella ty phi. Vaccine. 9:810-816.

23. Sundstrom, C., and K. Nilsson. 1976. Establishment and characterization of a human histiocytic lymphoma cell line (U937). Int. J. Cancer. 17:565-577.

24. Buchmeier, N. A., and F. Heffron. 1989. Intracellular survival of wild-type Salmonella typhimurium and macrophage-sensitive mutants in diverse populations of macrophages. Infect. Immun. 57:1-7.

25. Van De Verg, L., D. A. Herrington, J. R. Murphy, S. S. Wasserman, S. B. Formal, and M. M. Levine. 1990. Specific immunoglobulin A-secreting cells in peripheral blood of humans following oral immunization with a bivalent Salmo nella typhi-Shigella sonnei vaccine or infection by pathogenic $S$. sonnei. Infect. Immun. 58:2002-2004.

26. Wong, K. H., and J. C. Feeley. 1972. Isolation of Vi antigen and a simple method for its measurement. Appl. Microbiol. 24:628-633.

27. Ikeda, T., R. Kamiya, and S. Yamaguchi. 1983. Excretion of flagellin by a short-flagella mutant of Salmonella typhimurium. J. Bacteriol. 153:506-510.

28. Levine, M. M., C. Ferruccio, R. E. Black, C. O. Tacket, R. Germanier, and the Chilean Typhoid Committee. 1989. Progress in vaccines against typhoid fever. Rev. Infect. Dis. 11 (Suppl. 3):S552-S567.

29. Levine, M. M., H. L. DuPont, R. B. Hornick, M. J. Snyder, W. Woodward, R. H. Gilman, and J. P. Libonati. 1976. Attenuated, streptomycin-dependent Salmonella typhi oral vaccine: potential deleterious effects of lyophilization J. Infect. Dis. 133:424-429.

30. Hornick, R. B., S. E. Greisman, T. E. Woodward, H. L. Du Pont, A. T. Dawkins, and M. J. Snyder. 1970. Typhoid fever: pathogenesis and immunological control. N. Engl. J. Med. 283:686-691.

31. Gilman, R. H., R. B. Hornick, W. E. Woodward, H. L. DuPont, M. J. Snyder, M. M. Levine, and J. P. Libonati. 1977. Evaluation of a UDP-glucose-4 epimeraseless mutant of Salmonella typhi as a live oral typhoid vaccine. J. Infect. Dis. 136:717-723.

32. Snyder, M. J., R. B. Hornick, F. R. McCrumb, L. J. Morse, and T. E. Woodward. 1963. Asymptomatic typhoidal bacteremia in volunteers. Antimicrob. Agents Chemother. 7:604-607.

33. Dragunsky, E. M., E. Rivera, H. D. Hochstein, and I. S. Levenbook. 1990 In vitro characterization of Salmonella typhi mutant strains for live oral vaccines. Vaccine. 8:263-268.

34. Stocker, B. A. D., and H. Makela. 1986. Genetic determinants of bacterial virulence, with special reference to Salmonella. Curr. Top. Microbiol. Immunol. 124:149-172.

35. Milhous, W. K., N. F. Weatherly, J. H. Bowdre, and R. E. Desjardins. 1985. In vitro activities of an mechanisms of resistance to antifolate antimalarial drugs. Antimicrob. Agents Chemother. 27:525-530.
36. Hoiseth, S. 1983. PhD. Thesis. Stanford University, Palo Alto, CA.

37. Benjamin, W. H., C. L. Turnbough, B. S. Posey, and D. B. Briles. 1985. The inability of Salmonella typhimurium to produce siderophore enterobactin is not a virulence factor in mouse typhoid. Infect. Immun. 50:392-397.

38. Olitzki, A. 1972. The natural resistance to infections caused by enteric pathogens. In Enteric Fevers: Causing Organisms and Host's Reactions. S. Karger, New York. 78-182.

39. Greisman, S. E., R. B. Hornick, H. N. Wagner, W. E. Woodward, and T. E. Woodward. 1969. The role of endotoxin during typhoid fever and tularemia in man. IV. The integrity of the endotoxin tolerance mechanisms during infection. J. Clin. Invest. 48:613-629.

40. Tully, J. G., S. Gaines, and W. D. Tigertt. 1965. Studies on infection in experimental typhoid fever. VI. Response of chimpanzees to endotoxin and the effect of tolerance on resistance to oral challenge. J. Infect. Dis. 115:445-455.

41. Kelly, S., and R. Curtiss III. 1987. Salmonella typhimurium deletion mutants lacking adenylate cyclase and cyclic AMP receptor protein are avirulent and immunogenic. Infect. Immun. 55:3035-3043.

42. Emmerson, P. T., and S. C. West. 1977. Identification of protein X of Escherichia coli as the $\mathrm{recA}^{+} /$tif $^{+}$gene product. Mol. Gen. Genet. 155:77-85.

43. Miller, S. I., A. M. Kukral, and J. Mekalanos. 1989. A two-component regulatory system ( $p h o P$ phoQ) controls Salmonella virulence. Proc. Natl. Acad. Sci. USA. 86:5054-5058.

44. Miller, S. I., and J. J. Mekalanos. 1990. Constitutive expression of the PhoP regulon attenuates Salmonella virulence and survival within macrophages. J. Bacteriol. 172:2485-2490.

45. Galan, J. E., and R. Curtiss III. 1989. Virulence and vaccine potential of phoP mutants of Salmonella typhimurium. Microbial Pathol. 6:433-443.

46. Johnson, K., I. Charles, G. Dougan, D. Pickard, P. O’Gaora, G. Costa, T. Ali, I. Miller, and C. Hormaeche. 1991. The role of a stress-response protein in Salmonella typhimurium virulence. Mol. Microbiol. 5:401-407.

47. Buchmeier, N. A., and F. Heffron. 1990. Induction of Salmonella stress proteins upon infection of macrophages. Science (Wash. DC). 248:730-732.

48. Felix, A., S. S. Bhatnager, and R. M. Pitt. 1934. Observations on the properties of the Vi antigen of Bacillus typhosus. Br. J. Exp. Pathol. 15:346-354.

49. Johnson, F. M., B. Krauskopf, and L. S. Baron. 1965. Genetic mapping of Vi and somatic determinants in Salmonella. J. Bacteriol. 90:302-308.

50. Felix, A. and R. M. Pitt. 1951. The pathogenic and immunogenic activities of Salmonella typhi in relation to its antigenic constituents. J. Hyg. 49:92110.

51. Tacket, C. O., G. Losonsky, D. N. Taylor, L. S. Baron, D. Kopecko, S. Cryz, and M. M. Levine. 1991. Lack of immune response to the Vi component of a Vi-positive variant of the Salmonella typhi live oral vaccine strain Ty21a in human studies. J. Infect. Dis. 163:901-904.

52. Losonsky, G. A., C. Fereccio, K. L. Kotloff, S. Kaintuck, J. B. Robbins, and M. M. Levine. 1987. Development and evaluation of an enzyme-linked immunosorbent assay for serum Vi antibodies for detection of chronic Salmonella typhi carriers. J. Clin. Microbiol. 25:2266-2269.

53. Acharya, I. L., C. U. Lowe, R. Thapa, V. L. Gurubacharya, M. B. Shretha, M. Cadoz, D. Schulz, J. Armand, D. A. Bryla, B. Trollfors, et al. 1987. Prevention of typhoid fever in Nepal with the Vi capsular polysaccharide of Salmonella typhi. N. Engl. J. Med. 317:1101-1104.

54. Tacket, C. O., B. Forrest, R. Morona, S. R. Attridge, J. LaBrooy, B. D. Tall, M. Reymann, D. Rowley, and M. M. Levine. 1990. Safety, immunogenicity, and efficacy against cholera challenge in humans of a typhoid-cholera hybrid vaccine derived from Salmonella typhi Ty21a. Infect. Immun. 58:1620-1627.

55. Kantele, A., and P. H. Makela. 1991. Different profiles of the human immune response to primary and secondary immunization with an oral Salmonella typhi Ty21a vaccine. Vaccine. 9:423-427.

56. Kantele, A., J. M. Kantele, H. Arvilommi, and P. H. Makela. 1991. Active immunity is seen as a reduction in the cell response to oral live vaccine. Vaccine. 9:428-431. 